\title{
Quasispecies of Hepatitis B Virus
}

\author{
Jun Cheng, Min Quan, Min Li, Shun-ai Liu and Qi Wang
}

Hepatitis B virus (HBV) circulates in blood and replicates in the presence of quasispecies. During HBV replication, HBV DNA polymerase lacks fidelity and proofreading function partly because its exonuclease activity is either absent or deficient. Therefore, HBV genome is mutated with unusually high frequency. And these mutations can affect more than one open reading frame due to overlapping genes. Otherwise, natural substitutions, deletions or insertions involving the Cp/EN II locus in the X gene can significantly alter the extent of viral replication activity. Particular selection pressures such as host immune system and antiviral therapy readily select out escape mutants from this pre-existing quasispecies pool. Antiviral drug resistance in chronic hepatitis $\mathrm{B}(\mathrm{CHB})$ can be caused by the viral mutation frequency, the intrinsic mutability of the antiviral target site, the selective pressure exerted by the drug, the magnitude and rate of virus replication, the overall replication fitness of the mutant, the genetic barrier of the compound and the availability of replication space. Potent inhibition of HBV replication could be able to prevent the development of drug resistance because mutagenesis is replication dependent. Viral load may decline to a point where the continued production of quasispecies with the potential to resist new drug treatments no longer occurs, if viral replication can be suppressed for a sufficient length of time.

Key words: Quasispecies; Hepatitis B virus; Mutation

$\mathrm{H}$ epatitis B virus (HBV) circulates in blood and replicates in liver, as closely related, but genetically diverse molecules called quasispecies. ${ }^{1}$ For each patient, there is a viral reservoir with minor differences in sequence, the minority and majority of virus are subjected for evolution all the time. ${ }^{2}$ This is because during the replication, HBV production may approach $10^{11}$ molecules per day, although it may increase by 100-1000 times during peak activity. ${ }^{3}$ The two key events in the life-cycle of HBV involve: the generation from viral genomic DNA of the covalently closed circular DNA (cccDNA) transcriptional template, and the reverse transcription of the viral pregenomic RNA to form the HBV DNA genome. ${ }^{4}$ Generally, DNA polymerases have excellent fidelity in reading DNA templates because they are associated with an exonuclease which removes incorrectly added nucleotides. ${ }^{5}$ However, the HBV DNA polymerase lacks fidelity and proofreading function partly because exonuclease activity is either absent or deficient. ${ }^{6}$ Thus, the HBV genome is mutated with unusually high frequency. These mutations can affect more than one open reading frame because of overlapping genes. ${ }^{7}$

Natural substitutions, deletions or insertions

Correspondence: Institute of Infectious Diseases, Beijing Ditan Hospital, Capital Medical University

No. 8 East Jingshun Street, Chaoyang District, Beijing 100015, China. Tel: +86-10-84322006, Fax: +86-10-84397196; E-mail: jun.cheng. ditan@gmail.com involving the $\mathrm{Cp} / \mathrm{EN}$ II locus in the $\mathrm{X}$ gene can significantly alter the extent of viral replication activity. Particular selection pressures such as host immune system and antiviral therapy readily select out escape mutants from this pre-existing quasispecies pool. ${ }^{8}$ Antiviral drug resistance in chronic hepatitis B (CHB) can be caused by many factors, including the viral mutation frequency, the intrinsic mutability of the antiviral target site, the selective pressure exerted by the drug, the magnitude and rate of virus replication, the overall replication fitness of the mutant, the genetic barrier of the compound and the availability of replication space. ${ }^{9}$

Although the probability of developing viral resistance is directly proportional to the intensity of selection pressure and the diversity of quasispecies, potent inhibition of HBV replication should be able to prevent development of drug resistance because mutagenesis is replication dependent. If viral replication can be suppressed for a sufficient length of time, viral load should decline to a point where the continued production of quasispecies with the potential to resist new drug treatments no longer occurs. ${ }^{10}$

\section{EPIDEMIOLOGICAL IMPLICATIONS OF HBV QUASISPECIES}

Intrauterine transmission of $\mathrm{HBV}$ is one of the main reasons for the failure of vaccination and plays an 
important role in areas with high HBV prevalence. HBV quasispecies analysis is a powerful tool for the study on HBV transmission routes. Sun et al isolated HBV sequences from pairs of HBsAg-positive mothers and their neonates, who were infected with HBV by intrauterine transmission. ${ }^{11}$ Phylogenetic trees of the HBV strains of each pair was constructed, the topological structures were compared, and the distance between and within the quasispecies was calculated. From the phylogenetic trees, four types of HBV quasispecies has been identified. In the first type, the maternal and neonatal sequences clustered into one clade. In the second type, the sequences of the mothers and neonates formed separate monophyletic clusters, and the two clades were sister groups. In the third type, the strains of mother were the ancestors of the neonatal strains. In the fourth type, the strains of the mothers clustered with only some of the sequences of the neonate, and the other strains of the neonate formed another monophyletic group indicating a rapid HBV replication and mutation in the neonate bodies.

From the quasispecies analysis, the epidemic history and evolutionary dynamics of HBV infection could be traced. Forbi et al analyzed HBV infection in two remote communities in rural Nigeria, which were hyperendemic with unknown nature and origin. ${ }^{12}$ In this two semi-isolated rural communities in Nigeria, approximately $11 \%$ of the entire population was HBV DNA seropositive. Phylogenetic analysis showed extensive intermixing between $\mathrm{HBV} / \mathrm{E}$ variants identified in these communities and different countries in Africa. Quasispecies analysis showed extensive intra-host heterogeneity and inter-host variant sharing. The time to the most recent common ancestor (tMRCA) was estimated to be year 1952. Using additional $\mathrm{HBV} / \mathrm{E}$ sequences from other African countries, the tMRCA was estimated to be year 1948, indicating that $\mathrm{HBV} / \mathrm{E}$ in these remote communities has a similar time of origin with multiple HBV/E variants broadly circulating in West/Central Africa. Phylogenetic analysis and statistical neutrality tests suggested rapid HBV/E population expansion. Additionally, skyline plot analysis showed an increase in the size of HBV/ E-infected population over the last approximately $30-40$ years.

Xia et al determined the frequency of HBsAg mutations in the ' $\mathrm{a}$ ' determinant region among children who developed chronic HBV infection after receiving only active postexposure immunoprophylaxis. ${ }^{13}$ The most frequent amino acid substitutions observed were at residues 145, 126 and 133. However, there was no difference in the prevalence of 145 and 126 amino acid mutants between carrier cases and controls. The prevalence of $145 \mathrm{Arg}$ and 145 Ala mutants that were detected at similar levels among carrier cases, women controls, and children controls. The results showed that HBV mutants in the determinant were fairly consistent observed but without immune selective pressures. HBV variant strains may pre-existent as minor quasispecies. The prevalence of mutants is related to HBV subtypes and genotypes.

The immunoassay for HBV antigen and antibody should be affected by evolution of HBV quasispecies. The $\mathrm{S}$ gene contains an exposed major hydrophilic region (residues 110-155), which encompasses the 'a' determinant that is important for inducing immunity. Nucleotide substitutions in this region are common and result in reduced binding or failure to detect HBsAg in diagnostic assays. Adaptive immunity also depends on the recognition of HBsAg by specific antibody and variants pose a threat if they interfere with binding to antibody. Finally, genomic hypervariability allows HBV to escape selection pressures imposed by antiviral therapies, vaccines and host immune system, and is responsible for creating genotypes, subgenotypes and subtypes. $^{14}$

\section{HBV QUASISPECIES AND IMMUNE SELECTION}

The existence of HBV as quasispecies is thought to be favored by the infidelity of HBV RT, which would account for the emergence of many natural mutants with point substitutions. Some of naturally occurred mutation could not escape from host immune selection pressure and die eventually. So the living HBV mutants are all tolerant to the host immune pressure. The fitness of HBV mutant was determined by both HBV itself and immune pressure for each viral group. HBV infection is a dynamic process during which molecular variants are selected continuously to adapt to changes. Considerable evidence suggested that immune mechanisms were involved in the pathogenesis of HBV infection. ${ }^{15}$

Tsai et al demonstrated that both class I -restricted $\mathrm{CD} 8^{+} \mathrm{T}$ cell and class II -restricted $\mathrm{CD} 4^{+} \mathrm{T}$ cell responses to viral antigens were important mechanisms that may be responsible for the hepatocytes damage in hepatitis B. ${ }^{16} \mathrm{CD}^{+} \mathrm{T}$ cell proliferative responses to $\mathrm{HBcAg}$ are correlated with hepatitis activity. The responses existed in both adult and pediatric patients, and are more vigorous in patients with acute selflimited hepatitis B than in patients with CHB. In terms of major histocompatibility complexes (MHC) class I -restricted, $\mathrm{CD} 8^{+}$cytotoxic $\mathrm{T}$ lymphocyte $(\mathrm{CTL})$ response, antigenic peptides derived from $\mathrm{HBcAg}$, $\mathrm{HBsAg}$, and polymerase have been demonstrated to be the targets for CTL recognition in hepatitis B patients. Multiple CTL epitopes within HBsAg, HBcAg and polymerase can be detected by sensitizing target cells with synthetic peptides. 
Acute exacerbations in $\mathrm{HBeAg}$ negative patients with chronic HBV infection are invariably associated with concurrent increases in the index of IgM class antibodies against the core protein (anti-HBc) of the virus. Alexopoulou et al investigated whether anti$\mathrm{HBc}-\operatorname{IgM}$ response was related to the clearance of variants from the quasispecies pool, with amino acid substitutions in well recognized B-cell epitopes. ${ }^{17}$ The sequences at different time points were compared with those from a single $\mathrm{HBeAg}$ positive patient with no apparent acute exacerbations. Sequence comparison results showed that virus variants emerged in all, but two subsequent sera with amino acid substitutions affecting B-cell epitopes. It is concluded that the rise in the values of $\operatorname{IgM}$ anti-HBc may be attributed to the alteration of the antigenic epitopes leading to new antibody production in the majority of the cases.

Besides immune selection, antiviral therapy is also involved in the selection processes, especially sequential antiviral therapy may also lead to the selection of deleted mutants. Ji et al studied on one patient with lamivudine therapy failure and then switched to adefovir dipivoxil. ${ }^{18}$ Sequence analysis of RT region showed that the prevailing lamivudine resistant mutations were reduced after switching to adefovir dipivoxil, and ultimately the mutations were undetectable. Quasispecies distribution and deletion patterns in the $\mathrm{C}$ and pre-S regions were also different between the two periods of antiviral therapies. In lamivudine-treated samples, wild-type strains were dominant and deletions in the pre-S region were observed. However, after switching to adefovir dipivoxil, a virus population harboring deletions in $\mathrm{C}$ gene prevailed. Both major deletions encompassed T- and B-cell epitopes. Meanwhile, the frequencies of pre-S deletions decreased significantly, except a $129 \mathrm{bp}$ deletion. The presence deletions were always accompanied with some nucleotide substitutions. The prevalence of deletions at $\mathrm{C}$ gene epitopes accompanied with the gradual disappearance of lamivudine resistance mutations may contribute to the survival of HBV under sequential antiviral therapy.

\section{HBV QUASISPECIES IN OCCULT HBV INFECTION}

Occult hepatitis B virus infection (OBI) is defined as the presence of HBV DNA without detectable HBsAg in the serum. The prevalence of OBI was $1.5 \%$ to $1.7 \%$ in HBV infected population. OBI is more prevalent during HBV/HIV co-infection. Mutations occurring at locations of $\mathrm{Cp} / \mathrm{EN} \mathrm{II}$, and at B-cell epitope sites of S gene are associated with failure to detect serological markers of $\mathrm{HBV}$ infection. HBV variation can also arise from recombination between co-infected strains. ${ }^{19}$
S gene mutations that become evident following HBIG administration and HBV vaccination are all point substitutions, as are mutations in functional RT domains of the P gene after treatment with viral RTinhibitory drugs. Widespread and long-term use of prophylactic and therapeutic agents may potentially generate serologically occult $\mathrm{HBV}$ variants that might become difficult to eradicate. ${ }^{20}$

Genome sequence of HBV from OBI is scarce. In a cohort of 591 patients with chronic liver disease, Chaudhuri et al found 9.4\% seronegative for HBsAg and positive for HBV DNA. ${ }^{21}$ All patients with OBI except one had a low viral titer. Eight patients were infected with HBV genotype A and 6 with genotype D. Two patients infected with HBV genotype A had the characteristic nucleotide deletions in core and pre-S1 region seen in genotype $\mathrm{D}$. Whole genome sequence comparison indicated that frequent quasispecies variation, deletions in pre-S2/S region affecting the surface promoters and pre-S protein, truncated precore and core owing to stop signal, alternate start codon for the polymerase gene, and YMDD mutation in patients not on antiviral therapy. HBsAg and core proteins could be shown immunohistochemically in 3 of 5 liver biopsy specimens available. The mutant surface promoters (pre-S2 and S) on functional analysis showed alterations in HBsAg expression.

Zahn et al evaluated 9 of 32 OBI carriers with HBV serology, viral load and complete HBV genome sequences. ${ }^{22}$ All samples except one were anti-HBcpositive and three contained anti-HBs. All strains were of genotype $\mathrm{E}$ and formed quasispecies with $0.20 \%-1.28 \%$ intra-sample sequence variation. Few uncommon mutations were found across the entire genome. Two mutations in the core region encoded truncated or abnormal capsid protein, potentially affecting viral production, but were probably rescued by non-mutated variants, as found in one clone. No evidence of escape mutants was found in anti-HBscarrying samples, as the 'a' region was consistently wild type. OBI carriers appear as a disparate group reflecting decades of natural evolution in an area essentially devoid of human intervention. Martin et al amplified pre-S and S regions from OBI carriers. ${ }^{23} \mathrm{HBV}$ genotype was determined by phylogenetic analysis, while quasispecies diversity was quantified for each region. Sequence analysis identified new OBI mutations. Several of these OBI mutations likely contribute to the lack of detectable HBsAg in OBI infection by interfering with detection in serologic assays, altering antigen secretion and/or decreasing replication fitness. ${ }^{24}$

\section{COINFECTION WITH HIV AFFECTS HBV QUASISPECIES}

In the setting of HIV coinfection, the rate of HBV 
replication is increased by one to two orders of magnitude, accelerating the emergence of drug resistance in this situation. These HBV isolates with altered surface antigens exhibit reduced binding of specific and neutralizing antibody and so have diagnostic and public health implications, especially in the setting of HIV co-infection where the risk of transmission is increased. ${ }^{25}$

Compared with drug resistance rate observed during monotherapy of HIV infection, resistance is slow to emerge during treatment of hepatitis B. The rate of emergence might be dependent on the rate of infected hepatocyte turnover, which is extremely variable in chronic HBV infection (and significantly slower than infected lymphocyte turnover during HIV infection). Preliminary data suggested that pretreatment serum virus titre may be an important predictor of the development of drug resistance, an observation consistent with preexistance of the resistant virus in HBV quasispecies. ${ }^{26}$ HIV infection has a significant impact on the natural progression of liver disease caused by infection with $\mathrm{HBV}$, but its role in the molecular evolution of HBV is unknown. It is difficult to study the molecular evolution of HBV longitudinally considering its genomic complexity, which implies the analysis of paired samples.

Cassino et al analyzed the difference in the evolutionary dynamics of HBV among patients with or without HIV coinfection. ${ }^{27}$ The HBV phylogenetic inference among isolates from patients infected with HBV and coinfected with HBV and HIV tends to cluster separately. Likewise, when compared with the HBV evolutionary rate and genetic distances, values were higher in the former in both pre-C/C and $\mathrm{S}$ genomic regions. Intra-host analysis on HBV isolates revealed high complexity and diversity of quasispecies among patients infected with HBV exhibiting high numbers of viral variants and genetic distance. HBV exhibited low quasispecies complexity and diversity as well as low evolutionary rates in the presence of HIV coinfection, suggesting that coinfection may have an impact on the HBV molecular evolution most likely from the weakened cellular immune response.

The presence of HBV genomes with deletions at the $\mathrm{BCP}$ is associated with more aggressive liver disease. In the 3-year longitudinal analysis of two HIV/HBVcoinfected patients, Cassino et al identified three deletions with dissimilar abundance and permanence into the HBV quasispecies composition. ${ }^{28}$ These deletions may contribute to HBV pathogenesis in HIVcoinfected individuals. Cassino et al analyzed the role of genomic heterogeneity in $\mathrm{BCP}$ and precore (pre-C) genomic regions of $\mathrm{HBV}$ in $\mathrm{HBV} / \mathrm{HIV}$-coexistence. ${ }^{29}$ $\mathrm{BCP}$ mutations in isolates from monoinfected patients were significantly more frequent than in those from coinfected ones, irrespective of the $\mathrm{HBeAg}$ expression pattern. Regardless of the HIV-coexistence, the pre-C mutation at G1896A only barely appeared among clone-derived sequences of GtF1 isolates, mainly from HBeAg (-) HBV-monoinfected patients. HBV isolates characterized from HIV-coinfected patients seem to be more prone to exhibit a wild type genomic pattern at $\mathrm{BCP}$ regulatory region with respect to those from HBVmonoinfected ones. Besides, mutations at pre-C region might be genotype-dependent in their frequency but not on HIV co-presence related.

Lada et al evaluated quasispecies variability and in vitro drug susceptibility of good and delayed responders to tenofovir dipivoxil treatment of HBV/ HIV coinfected patients. ${ }^{30}$ Delayed responders, compared with good responders, showed a higher incidence of lamivudine-resistant mutations and a higher proportion of HBV genotype G. Clonal analysis demonstrated a higher variability of HBV quasispecies in delayed reponders than in good responders. In vitro analysis showed a lower efficacy of adefovir dipivoxil and tenofovir dipivoxil in delayed reponders. Furthermore, HBV genotype G strains showed a mild to weak susceptibility to tenofovir dipivoxil. Delayed responders showed higher quasispecies variability, a higher proportion of HBV genotype $\mathrm{G}$ and a mild in vitro decreased susceptibility to tenofovir dipivoxil and adefovir dipivoxil. A combination of these factors in heavily treatment-experienced HIV-infected patients could explain the lower tenofovir dipivoxil activity.

\section{HBV QUASISPECIES AND DISEASES PROGRESSION AND DETERIORATION}

Acute exacerbation of CHB is usually preceded by reemergence or increase of $\mathrm{HBV}$ in the serum. To investigate the origin of the reemergence or increase, Liu et al compared the identity of the serum viral genome to that in liver and in previous acute exacerbation. ${ }^{31}$ The viral variant that emerged after previous acute exacerbation was not identical to that caused by the subsequent acute exacerbation. The findings suggested that viral strain in serum reflected the intrahepatic strain of the acute exacerbation. Random reactivation of the original HBV pool, rather than a sequential evolution of one strain, also contributes to the onset of repeated acute exacerbation. Liu et al found quasispecies complexity of RT regions was significantly lower at the points of exacerbations, than that at the points of resolutions. ${ }^{32}$ Ratio of dominant quasispecies in HBV population was significantly higher during exacerbations than that during resolutions. All dominant quasispecies, except the one during the second resolution, carried core P5T, L60V, S155T, and pre-C G1896A mutations. 
Zhang et al analyzed four CHB patients who developed resistance to lamivudine accompanied by acute exacerbation of disease. Isolates during exacerbation showed significant high replication competence compared with isolates prior treatment. ${ }^{33}$ Apart from mutations at the YMDD motif, no shared mutations were shown among all isolates. In patients with acute exacerbation, high replication strains might be selected from the total HBV quasispecies during treatment, and amongst these strains, those with core promoter mutations were most likely to be associated with severe clinical exacerbations. But Li et al found the nucleotide divergence rate and the composition of quasispecies in the HBV sequence at the time of acute exacerbation were almost the same as found at other time points. ${ }^{34}$ These results suggested that acute exacerbation does not appear to be caused by a characteristic HBV species. The multiple factors that cause generalized HBV replication activation may contribute to acute exacerbation.

\section{HBV QUASISPECIES EVOLUTION DURING ANTIVIRAL THERAPY}

To characterize the HBV genetic heterogeneity in association with anti-viral therapy, Nishijima et al performed ultra-deep sequencing of full-genome HBV in the liver and serum of CHB patients, including therapy-naïve and NA-treated cases. ${ }^{35}$ Most genomic changes observed in viral variants were single base substitutions and were widely distributed throughout the HBV genome. Therapy-naïve HBeAg-negative patients showed a relatively low prevalence of the G1896A pre-C mutant in liver tissues, suggesting that other mutations were involved in their $\mathrm{HBeAg}$ seroconversion. Interestingly, liver tissues of NAtreated anti-HBe-positive cases had extremely low levels of the G1896A pre-C mutant, suggesting the high sensitivity of the G1896A pre-C mutant to NA. Moreover, various abundances of clones resistant to NA were common in both liver and serum of treatmentnaïve patients, and the proportion of M204VI mutants resistant to lamivudine and entecavir expanded in response to entecavir treatment, suggesting the putative risk of developing drug resistance to NA.

Karatayli et al analyzed a patient with anti-HBepositive CHB who had received lamivudine treatment for 7 years following an initial unsuccessful interferon treatment. ${ }^{36}$ The therapy had been switched to adefovir and then to entecavir when breakthrough occurred during each treatment. Dominance of a clone carrying L80LV, L91I, M204I, S219A, N238D, Y245H changes was detected in the last serum sample of the patient just before his death. This pattern displayed 30.4 fold resistance to entecavir when compared with the wild type HBV by in vitro phenotyping assay.

To explore the evolutionary pattern of full HBV genome during sequential NA therapy, Tang et al studied the evolutionary and mutational patterns of full HBV quasispecies. ${ }^{37} \mathrm{Co}$-variations in the core promoter, the pre-C region and in the known epitopes of the pre-S gene accompanied by RT mutations were common. In untreated patients, most co-variations located in the pre-C/C gene. In conclusion, the distribution of genetic variability of HBV shows remarkably different patterns between the treated and untreated subjects and the quasispecies divergence of different regions of HBV may vary remarkably even within a single host.

Liu et al found that quasispecies complexity and diversity were comparable between responders and partial responders to entecavir therapy at baseline. ${ }^{38}$ However, quasispecies complexity in responders at week 4 was statistically lower than that in partial responders at the nucleotide sequence level. Net changes in diversity as well as the viral nucleotide substitution rate of responders were higher than those of partial responders, and both correlated with virological responses. A preliminary model of quasispecies evolution variables predicted responders and partial responders in the entecavir group. Despite significant differences between responders to entecavir and responders to lamivudine at week 4, the characteristics of quasispecies were quite similar between partial responders to entecavir and nonresponders to lamivudine.

Chen et al also demonstrated the quasispecies complexity and diversity were not different between responders and non-responders to lamivudine therapy at baseline. ${ }^{39}$ However, the quasispecies complexity and diversity of responders were significantly lower than those of non-responders at week 4. Furthermore, the viral nucleotide substitution rate of responders was significantly higher than that of non-responders. The dynamic changes of HBV quasispecies within the RT region showed distinct patterns between responders and non-responders during early stage of lamivudine treatment. ${ }^{40}$ The dynamic changes of quasispecies complexity and diversity during the first 4 weeks were correlated with lamivudine antiviral efficacy and antiviral resistance.

\section{REFERENCES}

1. Jazayeri SM, Alavian SM, Carman WF. Hepatitis B virus: origin and evolution. J Viral Hepat 2010;17:229-235.

2. Yim HJ. Hepatitis B virus genetic diversity and mutant. Korean J Hepatol 2008;14:446-464.

3. Sheldon J, Rodès B, Zoulim F, Bartholomeusz A, Soriano V. Mutations affecting the replication capacity of the hepatitis B virus. J Viral Hepat 2006;13:427-434.

4. Bartholomeusz A, Locarnini S. Hepatitis B virus mutations associated with antiviral therapy. J Med Virol 2006;78 Suppl 
1:S52-55.

5. Osiowy C. Detection of HBsAg mutants. J Med Virol 2006;78 Suppl 1:S48-S51.

6. Durantel D, Brunelle MN, Gros E, Carrouée-Durantel S, Pichoud $\mathrm{C}$, Villet S, et al. Resistance of human hepatitis B virus to reverse transcriptase inhibitors: from genotypic to phenotypic testing. J Clin Virol 2005;34 Suppl 1:S34-43.

7. Pawlotsky JM. The concept of hepatitis B virus mutant escape. J Clin Virol 2005;34 Suppl 1:S125-129.

8. Lee JY, Locarnini S. Hepatitis B virus: pathogenesis, viral intermediates, and viral replication. Clin Liver Dis 2004;8:301-320.

9. Durantel D. Fitness and infectivity of drug-resistant and crossresistant hepatitis B virus mutants: why and how is it studied? Antivir Ther 2010;15:521-527.

10. Lee JY, Locarnini S. Hepatitis B virus: pathogenesis, viral intermediates, and viral replication. Clin Liver Dis 2004;8:301-320.

11. Sun H, Tang X, Wang B, Zhang J, Cheng H, Su H, et al. A preliminary study on the molecular evolution of the two routes of intrauterine transmission of HBV. Arch Virol 2012;157:155-164.

12. Forbi JC, Vaughan G, Purdy MA, Campo DS, Xia GL, GanovaRaeva LM, et al. Epidemic history and evolutionary dynamics of hepatitis B virus infection in two remote communities in rural Nigeria. PLoS One 2010;5:e11615.

13. Xia G, Nainan OV, Jia Z, Wang JJ, Liu HB, Li RC, et al. Prevalence of mutants in the determinant region of hepatitis $\mathrm{B}$ surface antigen among Chinese carriers after receiving only active postexposure immunoprophylaxis. Zhonghua Shi Yan He Lin Chuang Bing Du Xue Za Zhi 2001;15:212-215.

14. Hollinger FB. Hepatitis B virus genetic diversity and its impact on diagnostic assays. J Viral Hepat 2007;14 Suppl 1:11-15.

15. Alexopoulou A, Dourakis SP. Genetic heterogeneity of hepatitis viruses and its clinical significance. Curr Drug Targets Inflamm Allergy 2005;4:47-55.

16. Tsai SL, Huang SN. T cell mechanisms in the immunopathogenesis of viral hepatitis B and C. J Gastroenterol Hepatol 1997; 12:S227-235.

17. Alexopoulou A, Baltayiannis G, Eroglu C, Nastos T, Dourakis $\mathrm{SP}$, Archimandritis AJ, et al. Core mutations in patients with acute episodes of chronic HBV infection are associated with the emergence of new immune recognition sites and the development of high IgM anti-HBc index values. J Med Virol 2009;81:34-41.

18. Ji F, Zhou L, Ma S, Li F, Ding H, Zeng C. Dynamic changes of HBV quasispecies and deletion patterns in a chronic hepatitis B patient. J Med Virol 2009;81:1551-1559.

19. Zahn A, Li C, Danso K, Candotti D, Owusu-Ofori S, Temple J, et al. Molecular characterization of occult hepatitis B virus in genotype E-infected subjects. J Gen Virol 2008;89:409-418.

20. Ngui SL, Hallet R, Teo CG. Natural and iatrogenic variation in hepatitis B virus. Rev Med Virol 1999;9:183-209.

21. Chaudhuri V, Tayal R, Nayak B, Acharya SK, Panda SK. Occult hepatitis B virus infection in chronic liver disease: full-length genome and analysis of mutant surface promoter Gastroenterology 2004;127:1356-1371.

22. Zahn A, Li C, Danso K, Candotti D, Owusu-Ofori S, Temple J, et al. Molecular characterization of occult hepatitis B virus in genotype E-infected subjects. J Gen Virol 2008;89:409-418.

23. Martin CM, Welge JA, Shire NJ, Rouster SD, Shata MT, Sherman $\mathrm{KE}$, et al. Genomic variability associated with the presence of occult hepatitis B virus in HIV co-infected individuals. J Viral Hepat 2010;17:588-597.

24. Homs M, Buti M, Quer J, Jardí R, Schaper M, Tabernero D, et al. Ultra-deep pyrosequencing analysis of the hepatitis $\mathrm{B}$ virus
preCore region and main catalytic motif of the viral polymerase in the same viral genome. Nucleic Acids Res 2011;39:8457-8471.

25. Locarnini S, Warner N. Major causes of antiviral drug resistance and implications for treatment of hepatitis B virus monoinfection and coinfection with HIV. Antivir Ther 2007;12 Suppl 3:H15-23.

26. Mutimer D. Hepatitis B virus antiviral drug resistance: from the laboratory to the patient. Antivir Ther 1998;3:243-246.

27. Cassino L, Torres C, Mbayed V, Laufer N, Campos RH, Quarleri J. Comparative analysis of hepatitis B virus genotype a molecular evolution in patients infected with HBV and in patients coinfected with HBV and HIV. J Med Virol 2012;84:562-569.

28. Cassino L, Laufer N, Giuliano SF, Bouzas MB, Quarleri J. Hepatitis $\mathrm{B}$ virus harboring nucleotide deletions in the basal core promoter in HBe-positive HIV-coinfected patients under lamivudine therapy. AIDS Res Hum Retroviruses 2011;27:543-545.

29. Cassino L, Laufer N, Salomon H, Campos R, Quarleri J. Hepatitis B precore/core promoter mutations in isolates from HBV-monoinfected and HBV-HIV coinfected patients: a 3-yr prospective study. J Clin Virol 2009;46:354-359.

30. Lada O, Gervais A, Branger M, Peytavin G, Roquebert B, Collin $\mathrm{G}$, et al. Quasispecies analysis and in vitro susceptibility of HBV strains isolated from HIV-HBV-coinfected patients with delayed response to tenofovir. Antivir Ther 2012;17:61-70.

31. Liu CJ, Kao JH, Wang HY, Lai MY, Chen TC, Chen PJ, et al. Origin of serum hepatitis $B$ virus in acute exacerbation: comparison with $\mathrm{HBV}$ in the liver and from other exacerbation. Hepatology 2004;40:310-317.

32. Liu L, Wang YM, Lan L, Li JG, Deng GH, Xia J, et al. Study of the quasispecies dynamics of serum hepatitis $\mathrm{B}$ virus in a patient with acute exacerbations of chronic hepatitis B. Zhonghua Gan Zang Bing Za Zhi 2006;14:29-32.

33. Zhang JM, Yao X, Wang YX, Liu F, Ma ZM, Weng XH, et al. High replicative full-length lamivudine-resistant hepatitis $\mathrm{B}$ virus isolated during acute exacerbations. J Med Virol 2005;77:203-208.

34. Li W, Ikematsu H, Yamaji TK, Chong Y, Hayashi J, Kashiwagi S. Hepatitis B virus genomes of chronic hepatitis patients do not contain specific mutations related to acute exacerbation. Dig Dis Sci 2001;46:2104-2112.

35. Nishijima N, Marusawa H, Ueda Y, Takahashi K, Nasu A, Osaki $\mathrm{Y}$, et al. Dynamics of hepatitis B virus quasispecies in association with nucleos(t)ide analogue treatment determined by ultra-deep sequencing. PLoS One 2012;7:e35052.

36. Karatayli E, Karatayli SC, Cinar K, Gokahmetoglu S, Güven K, Idilman R, et al. Molecular characterization of a novel entecavir mutation pattern isolated from a multi-drug refractory patient with chronic hepatitis B infection. J Clin Virol 2012;53:130-134.

37. Tang YZ, Liu L, Pan MM, Wang YM, Deng GH. Evolutionary pattern of full hepatitis B virus genome during sequential nucleos(t)ide analog therapy. Antiviral Res 2011;90:116-125.

38. Liu F, Chen L, Yu DM, Deng L, Chen R, Jiang Y, et al. Evolutionary patterns of hepatitis $\mathrm{B}$ virus quasispecies under different selective pressures: correlation with antiviral efficacy. Gut 2011;60:1269-1277.

39. Chen L, Zhang Q, Yu DM, Wan MB, Zhang XX. Early changes of hepatitis B virus quasispecies during lamivudine treatment and the correlation with antiviral efficacy. J Hepatol 2009;50:895-905.

40. Lada O, Gervais A, Branger M, Peytavin G, Roquebert B, Collin $\mathrm{G}$, et al. Quasispecies analysis and in vitro susceptibility of HBV strains isolated from HIV-HBV-coinfected patients with delayed response to tenofovir. Antivir Ther 2012;17:61-70. 